07,14

\title{
Применение нелокальных критериев разрушения в задачах с неоднородным полем напряжений
}

\author{
(C) А.А. Чеврычкина, А.А. Груздков, Ю.В. Петров \\ Санкт-Петербургский государственный университет, \\ Санкт-Петербург, Россия \\ E-mail: Anastasiia.Che@gmail.com \\ (Поступила в Редакцию 22 декабря 2016 г. \\ В окончательной редакции 16 февраля 2017 г.)
}

\begin{abstract}
Рассматривается зависимость критической нагрузки от степени неоднородности поля напряжений на примере балок и пластин из полиметилметакрилата с круглым отверстием или угловым вырезом. Предлагается использовать нелокальные критерии хрупкого разрушения для описания поведения неоднородно напряженного материала. Среди рассмотренных критериев структурный критерий является наиболее универсальным, так как в этом случае параметр расстояния $d$ наименее зависит от схемы испытания. Параметры расстояния в задачах с регулярным концентратором имеют значения одного порядка, так же как и в задачах с сингулярным концентратором напряжений. Дальнейшая модификация критерия с учетом свойств процесса разрушения, возможно, позволит получить единое значение параметра размерности длины для любой степени неоднородности поля напряжений.
\end{abstract}

Работа выполнена при поддержке РФФИ (гранты № 16-51-53007, 17-01-00618), фонда Марии Кюри (TAMER N 610547) и программы № 25 Президиума РАН.

Раздел 3 выполнен Чеврычкиной А.А. при поддержке РНФ (грант № 17-11-01053).

DOI: $10.21883 /$ FTT.2017.08.44760.456

\section{1. Введение}

Условие прочности твердого тела в рамках классического подхода можно представить в виде

$$
f\left(\sigma_{i j}\right) \leq \sigma_{c},
$$

где $f\left(\sigma_{i j}\right)$ - некоторая функция компонент тензора напряжений $\sigma_{i j} ; \sigma_{c}$ - критическое напряжение материала. Наступлению предельного состояния (разрушению) соответствует знак равенства в выражении (1).

Экспериментальные данные показывают, что значение критического напряжения в критерии (1) зависит от степени неоднородности напряженного состояния материала. Например, при изгибе в материале могут достигаться напряжения, существенно превосходящие прочность материала [1,2]. В инженерной практике критическое напряжение, определяемое при изгибе, называют изгибной прочностью. Изгибная прочность входит в набор независимых прочностных параметров, который используется для описания поведения материала. Недостаток этого подхода состоит в том, что для разных типов схем испытаний приходится вводить разные характеристики прочности. Поэтому актуальными являются нелокальные критерии разрушения, которые позволяют охватывать поля напряжений разной степени неоднородности и с единых позиций подходить к оценке предельных состояний в задачах и с однородными, и с неоднородными полями напряжений.

В нелокальных критериях в отличие от классических разрушение рассматривают как физический процесс, происходящий в некоторой области, а не как событие достижения максимального напряжения в некоторой точке. Условие прочности удобно записать в виде

$$
f\left(\sigma_{i j}, \nabla \sigma, d, \sigma_{c}\right) \leq 1,
$$

где $f$ - некоторый функционал, $\sigma_{c}-$ критическое напряжение материала без макродефектов, $d$ - параметр размерности длины, $\nabla \sigma$ - градиент напряжений.

Предполагается, что для поставленных задач верна линейная механика разрушения, поэтому рассматриваются критерии, описывающие хрупкое разрушение. В работе проведен сравнительный анализ критериев НейбераНовожилова [3,4], Питерсона [4] и градиентного критерия, предложенного Харлабом [5], на основе сопоставления результатов расчета с экспериментальными данными.

\section{2. Нелокальные критерии}

2.1. Структурный критерий Нейбера-Новож и л о в а. Этот критерий учитывает напряженное состояние во всех точках, расположенных на отрезке длины $d$ от рассматриваемой точки в направлении вероятного распространения трещины.

Общую форму записи критерия удобно представить в полярной системе координат $(r, \theta)$

$$
\max _{-\pi<\theta \leq \pi} \int_{0}^{d} \sigma\left(\mathbf{y}_{0}+\boldsymbol{\eta}(\theta) r\right) d r=\sigma_{c} d .
$$

Начало полярной системы координат $(r, \theta)$ расположено в точке вероятного разрушения, задаваемой 


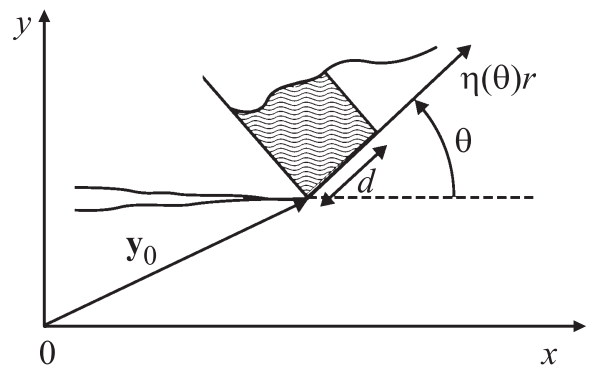

Рис. 1. Расположение систем координат и параметров расстояния в случае критерия Нейбера-Новожилова.

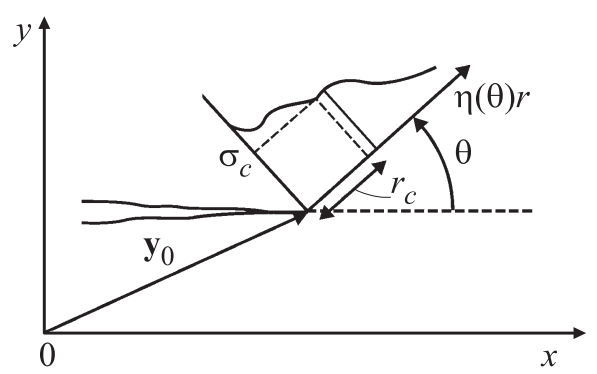

Pис. 2. Расположение систем координат и параметров расстояния в случае критерия Питерсона.

вектором $\mathbf{y}_{0}, \boldsymbol{\eta}(\theta)$ - единичный вектор, исходящий из начала координат полярной системы, - составляет угол $\theta$ с осью ординат (рис. 1).

Если известно, в каком направлении $\theta_{0}$ достигается максимум в формуле (2), то условие достижения предельного состояния в критерии можно представить в более простой форме

$$
\int_{0}^{d} \sigma(r) d r=\sigma_{c} d
$$

2.2. Критерий Питерсона. В 1930 г. Питерсон предложил критерий для определения критического состояния при усталостном нагружении тел с угловым вырезом. Данный подход также используется в предсказании хрупкого разрушения. Общий вид критерия при неизвестном направлении распространения трещины удобно представить в полярной системе координат

$$
\max _{-\pi<\theta \leq \pi}\left[\min _{0 \leq r \leq r_{c}} \sigma\left(\mathbf{y}_{0}+\boldsymbol{\eta} r\right)\right]=\sigma_{c} .
$$

Предполагается, что разрушение произойдет, если напряжение во всех точках отрезка $\left[0 ; r_{c}\right]$ в направлении $\theta=\theta_{0}$ превосходит $\sigma_{c}$, где $\theta=\theta_{0}$ - предполагаемое направление распространения трещины (рис. 2). Этот критерий хуже, чем предыдущий, учитывает особенности распределения напряжений на рассматриваемом участке, например выпуклость распределения напряжений.
Условие достижения предельного состояния при известном направлении $\theta=0$ примет вид

$$
\min _{0 \leq r \leq r_{c}} \sigma\left(\mathbf{y}_{0}+\boldsymbol{\eta}\left(\theta_{0}\right) r\right)=\sigma_{c} .
$$

Чаще всего наблюдается монотонное убывание напряжений при удалении от концентратора напряжений. В этом случае условие достижения предельного состояния принимает простой вид

$$
\sigma\left(r_{c}\right)=\sigma_{c} .
$$

2.3. Градиентный критерий Харлаба. Рассмотрим один из градиентных критериев прочности, а именно критерий, предложенный Харлабом. При выводе критерия автор исходил из того что критерий, учитывающий неоднородное напряженное состояние, должен включать в себя помимо характерного напряжения $\sigma$ величину его относительного изменения $\delta|\nabla \sigma| \sigma$ на характерной для материала длине $\delta$ так, чтобы неоднородность напряженного состояния повышала сопротивление материала разрушению:

$$
\max _{-\pi \leq \theta \leq \pi} \max _{0 \leq r \leq \delta} \frac{\sigma\left(\mathbf{y}_{0}+\boldsymbol{\eta}(\theta) r\right)}{1+\frac{\delta\left|\nabla \sigma\left(y_{0}+\boldsymbol{\eta}(\theta) r\right)\right|}{\sigma\left(\mathbf{y}_{0}+\boldsymbol{\eta}(\theta) r\right)}}=\sigma_{c},
$$

где $\mathbf{y}=\mathbf{y}_{0}+\boldsymbol{\eta}\left(\theta_{0}\right) r, \delta-$ параметр материала размерности длины, $\nabla-$ символ градиента.

Значения параметров $d, r_{c}, \delta$ можно определять из условия достижения предельного состояния соответствующих критериев в задачах с неоднородным распределением напряжений.

\section{3. Применение нелокальных критериев к задачам с неоднородным полем напряжений}

3.1. Растяжение пластины с круглым отверстием. Проанализируем с позиции нелокальных критериев экспериментальные данные по прочностным свойствам полиметилметакрилата из работы [6]. Прочность на растяжение рассматриваемого материала $\sigma_{c}=72 \mathrm{MPa}$. Рассмотрим разрушение при растяжении пластин с регулярным концентратором напряжений в виде центрального кругового отверстия (рис. 3). Распределение напряжений в образце можно оценить, воспользовавшись известным решением задачи Кирша [7], когда диаметр отверстия мал по сравнению с размерами пластины:

$$
\sigma_{y}(x)=\frac{p}{2}\left(2+\frac{a^{2}}{x^{2}}+3 \frac{a^{4}}{x^{4}}\right),
$$

где $p-$ приложенная нагрузка, $a-$ радиус отверстия. Максимальные напряжения имеют место на контуре отверстия в точках $x= \pm a$ и втрое превышают величину приложенного напряжения. 
Подставляем распределения напряжений (6) в формулы (2), (4), (5) и определяем, что критические нагрузки $p^{*}$, полученные на основе структурного критерия, критерия критической точки и градиентного критерия, равны соответственно

$$
\begin{gathered}
p^{*}=\sigma_{c}\left(1+\left(1+\frac{d}{a}\right)^{-1}+0.5\left(1+\frac{d}{a}\right)^{-2}\right. \\
\left.+0.5\left(1+\frac{d}{a}\right)^{-3}\right)^{-1}, \\
p^{*}=2 \sigma_{c}\left(2+\left(1+\frac{r_{c}}{a}\right)^{-2}+3\left(1+\frac{r_{c}}{a}\right)^{-4}\right)^{-1}, \\
p^{*}=\sigma_{c}\left(2+t^{2}+3 t^{4}\right)\left(2+\frac{\delta}{a} \frac{4 t^{3}\left(1+6 t^{2}\right)}{2+t^{2}+3 t^{4}}\right)^{-1},
\end{gathered}
$$

где $t=a / x^{*}, x^{*}$ - точка максимума по $x$ в правой части выражения (9).

Расчетная точка $x^{*}$ при $a / \delta \geq 1.638$ располагается на контуре отверстия $x^{*}=a$, при $a / \delta<1.638$ она смещается в глубь материала, так что область около отверстия становится как бы упрочненной. При анализе экспериментальных данных рассматривался только случай $a / \delta \geq 1.638$. В этом случае критическая нагрузка равна

$$
p^{*}=\frac{1}{3} \sigma_{c}\left(1+\frac{\delta}{a} \frac{7}{3}\right) .
$$

Экспериментальные данные и результаты расчета по формулам (7), (8), (10) со значениями параметров расстояния, подобранных методом наименьших квадратов, приведены на рис. 4. Представленные данные показы-

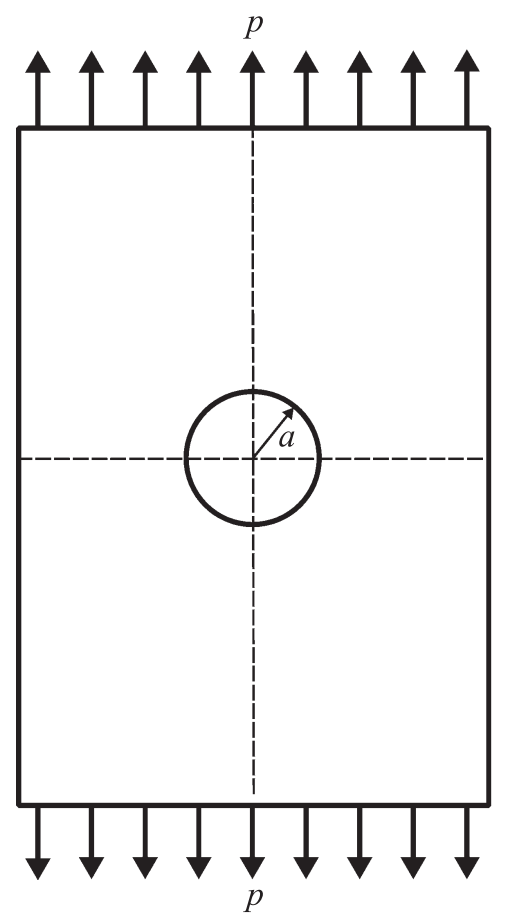

Рис. 3. Схематическое изображение растяжения пластины с центральным круглым отверстием.

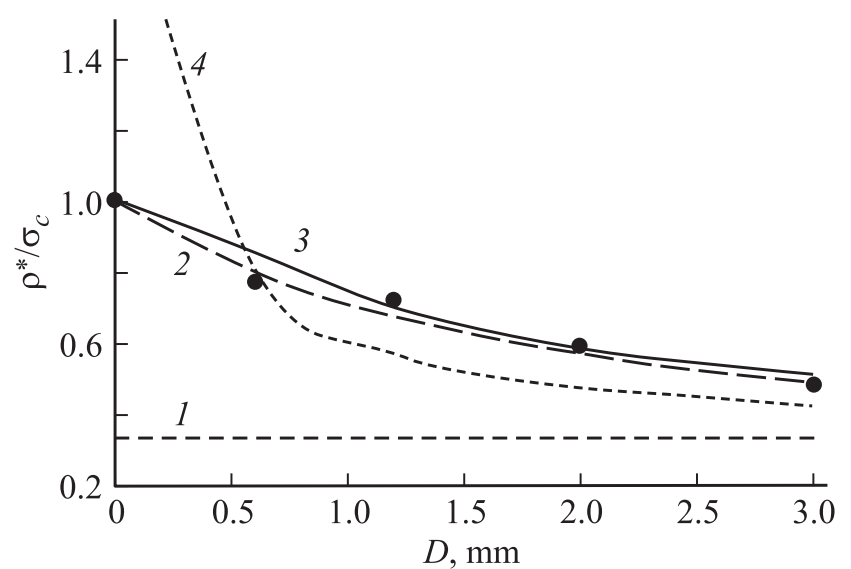

Рис. 4. Зависимость критического напряжения от диаметра отверстия ( $p^{*}-$ критическая нагрузка, $\sigma_{c}-$ прочность растяжения, $D-$ радиус отверстия). $1-$ классический критерий, 2 - критерий Нейбера-Новожилова, $d=1.1 \mathrm{~mm}, 3$ - критерий Питерсона, $r_{c}=0.36 \mathrm{~mm}, 4-$ критерий Харлаба, $\delta=0.21 \mathrm{~mm}$. Точками отмечены экспериментальные данные.

вают зависимость отношения критического напряжения к прочности материала от радиуса отверстия. Данный эффект достаточно хорошо описывается структурным критерием разрушения (2) и критерием критической точки (3).

Согласно приведенному графику, результат $p^{*} / \sigma_{c}=1 / 3$, вытекающий из классического условия разрушения $\max _{x} \sigma_{y}(x)=\sigma_{c}$, справедлив только для относительно больших отверстий $a / d, a / r_{c}, a / \delta \rightarrow \infty$. С уменьшением относительного радиуса отверстия $a / d$ или $a / r_{c}$ его влияние на прочность тела снижается и в пределе исчезает: $\lim _{a / d \rightarrow 0} p / \sigma_{c}=1$. Таким образом, подход структурного критерия и критерия критической точки устраняет вывод классической теории о трехкратном снижении прочности растягиваемой плоскости с отверстием сколь угодно малого радиуса. Значение критической нагрузки, полученной на основе градиентного критерия, возрастает с уменьшением радиуса и в пределе $a / \delta \rightarrow 0$ выходит на бесконечность, т.е. градиентный критерий Харлаба не устраняет дефекта классического критерия.

3.2. Растяжение пластины с угловым вырезом. Проанализируем с позиции нелокальных критериев экспериментальные данные о разрушении при растяжении пластин с угловым вырезом. В работе [6] приведены результаты испытаний пластин из полиметилметакрилата с угловыми вырезами, прочность на растяжение данного материала $\sigma_{c}=70.2 \mathrm{MPa}$. Распределение напряжений можно оценить, воспользовавшись решением задачи о растяжении пластины с угловым вырезом [8]. Формула

$$
\sigma_{\theta \theta, \theta=0}=p c(\omega)\left(\frac{r}{2 a}\right)^{\mu-1}
$$

определяет асимптотику напряженного состояния в окрестности угловой точки, когда в полярной системе 
координат направлению быстрейшего изменения напряжений соответствует $\theta=0$ (рис. 5). В формуле (11) $p$ - приложенное напряжение, $\mu, c(\omega)$ - некоторые коэффициенты, конкретные выражения можно найти в работе [8]. В вершине углового выреза в точке $r=0$ имеют место бесконечные напряжения.

Для угла раствора, равного $\omega=\pi / 2$, угловая точка в постановке задачи исчезает, задача сводится к растяжению „сплошной“ пластины. Формула (11) приобретает вид $\sigma_{\theta \theta, \theta=0}(\omega=\pi / 2)=3 p(\mu(\pi / 2)=1, c(\pi / 2)=3)$, что не соответствует случаю растяжения „сплошной“ пластины. Для угла раствора $\omega=\pi$ угловой вырез становится разрезом. Формула (11) со значением $\omega=\pi$ соответствует решению задачи о растяжении пластины с разрезом.

Область применения формулы (11) ограничивается следующим интервалом значений $\omega:(\pi / 2, \pi]$.

Подставляем распределение напряжений (11) в формулы (2), (4), (5) и определяем, что критические нагрузки $p^{*}$, полученные на основе структурного критерия, критерия критической точки и градиентного критерия, равны соответственно

$$
\begin{aligned}
& p^{*}=\sigma_{c} \frac{\mu 2^{\mu-1}}{c(\omega)}\left(\frac{d}{a}\right)^{1-\mu}, \\
& p^{*}=\sigma_{c}\left(\frac{r_{c}}{a}\right)^{1-\mu} \frac{2^{\mu-1}}{c(\omega)}, \\
& p^{*}=\sigma_{c}\left(\frac{\delta}{a}\right)^{1-\mu} \frac{2^{\mu-1}}{c(\omega)},
\end{aligned}
$$

где $\delta=(1-\mu) \delta$.

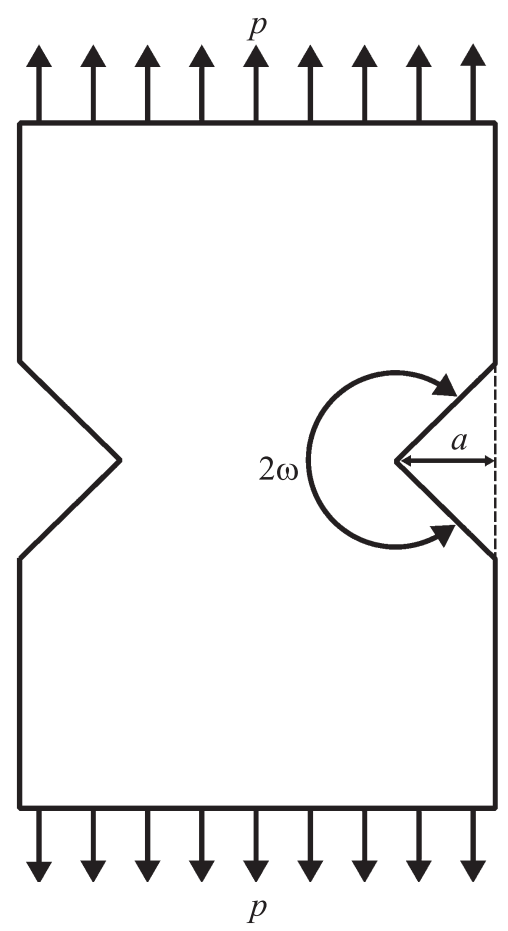

Рис. 5. Схематическое изображение растяжение пластины с угловыми вырезами.

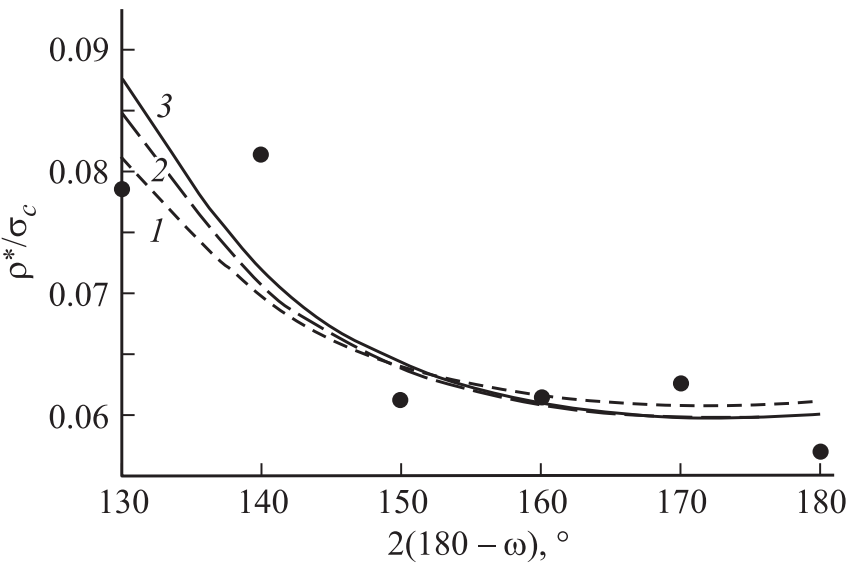

Рис. 6. Зависимость критической нагрузки от угла раствора $\left(360^{\circ}-2 \omega\right) .1-$ критерий Нейбера-Новожилова $d=0.18 \mathrm{~mm}$, 2 - критерий Питерсона $r_{c}=0.045 \mathrm{~mm}, 3$ - критерий Харлаба, $\delta=0.095 \mathrm{~mm}$. Точками отмечены экспериментальные данные.

Экспериментальные данные и результаты расчета по формулам (12)-(14) со значениями параметров расстояния, подобранных методом наименьших квадратов, приведены на рис. 6. Представленные данные демонстрируют зависимость критической нагрузки от угла раствора, которая довольно хорошо описывается всеми рассматриваемыми критериями разрушения.

3.3. Изгиб балки. Задача изгиба балок является интересным случаем неравномерного распределения напряжений. Предельное максимальное растягивающее напряжение балки, т.е. предел прочности при изгибе, возрастает с уменьшением толщины балки [2]. Распределение напряжений в поперечном сечении балки при плоском изгибе определяется следующими формулами, выведенными в предположении, что поведение материала балки упругохрупкое и выполняются гипотезы Бернулли:

$$
\sigma(y)=\sigma_{\max } \frac{2 y}{h}, \quad-\frac{h}{2} \leq y \leq \frac{h}{2},
$$

где $\sigma_{\max }=M h / 2 I_{x}-$ предельное максимальное растягивающее напряжение при изгибе, $I_{x}=b h^{3} / 12-$ момент инерции прямоугольного сечения балки, $M-$ момент относительно оси $O x$. Изгибающий момент равен $M=P L / 4$ в трехточечном изгибе.

Проанализируем с позиции нелокальных критериев экспериментальные данные для трехточечного изгиба балки. В работе [9] приведены данные о изгибе балки с параметрами $\sigma_{c}=72 \mathrm{MPa}, \sigma_{\max }=105 \mathrm{MPa}$, толщина балки $h=3 \mathrm{~mm}$.

3.3.1. Критическое условие структурного критерия (2) в задаче изгиба балки. Подставим распределение напряжений (15) в формулу (2) и выразим максимальное напряжение в поперечном сечении

$$
\sigma_{\max }^{*}=\frac{\sigma_{c}}{(1-d / h)} .
$$


Каждой толщине балки можно сопоставить такое значение расстояния $d$, при котором критическое напряжение, предсказанное структурным критерием, будет полностью совпадать с экспериментальными данными. Значение параметра $d$ в этом случае определяется формулой

$$
d=h\left(1-\frac{\sigma_{c}}{\sigma_{\max }}\right) .
$$

Максимальное нормальное напряжение в поперечном сечении превосходит прочность на растяжение, т.е. $\sigma_{c} / \sigma_{\max }<1$, и с уменьшением толщины балки возрастает из-за неоднородного распределения напряжений. Согласно формуле (16), структурный критерий с параметрами $\left(\sigma_{c}, d\right)$ прогнозирует, что балки с толщиной меньше $d$ не разрушатся ни при какой нагрузке, что невозможно. Структурный критерий прогнозирует корректный результат для балок с толщиной, большей $d$.

3.3.2. Критическое условие критерия критической точки (4) в задаче изгиба балки. Подставим распределение напряжений (15) в формулу (4) и выразим максимальное напряжение в поперечном сечении

$$
\sigma_{\max }^{*}=\frac{\sigma_{c}}{\left(1-2 r_{c} / h\right)}
$$

Каждой толщине балки можно сопоставить такое значение расстояния $r_{c}$, при котором напряжение, предсказанное критерием критической точки, будет полностью совпадать с экспериментальными данными. Значение $r_{c}$ определяется формулой

$$
r_{c}=\frac{h}{2}\left(1-\frac{\sigma_{c}}{\sigma_{\max }}\right) .
$$

Оценка толщин балок в критерии критической точки $\left(\sigma_{c}\right.$, $r_{c}$ ) также существует и имеет вид $h>2 r_{c}$. Критерий критической точки $\left(\sigma_{c}, r_{c}\right)$ предсказывает, что балка с толщиной, меньшей $r_{c}$, не разрушится ни при каком значении нагрузки.

3.3.3. Критическое условие градиентного критерия (5) в задаче изгиба балки. Подставим распределение напряжений (15) в формулу (5) и выразим максимальное напряжение в поперечном сечении

$$
\sigma_{\max }^{*}=\sigma_{c}(1+2 \delta / h) \text {. }
$$

Значение $\delta$ определяется формулой

$$
\delta=\frac{h}{2}\left(\frac{\sigma_{\max }}{\sigma_{c}}-1\right) .
$$

Градиентный критерий не подразумевает никаких ограничений для слишком „тонких“ или „толстых“ балок.

Значения характерных расстояний $d, r_{c}, \delta$, полученных на основе экспериментальных данных работы [9], приведены в таблице.
Характерные расстояний $d, r_{c}, \delta$ для задач с неоднородным полем напряжений

\begin{tabular}{c|c|c|c|c}
\hline $\begin{array}{c}\text { Характерное } \\
\text { расстояние, } \\
\text { mm }\end{array}$ & $\begin{array}{c}\text { Пластина } \\
\text { с трещиной } \\
{[6]}\end{array}$ & $\begin{array}{c}\text { Пластина } \\
\text { с вырезом }\end{array}$ & $\begin{array}{c}\text { Пластина } \\
\text { с круглым } \\
\text { отверстием }\end{array}$ & $\begin{array}{c}\text { Изгиб } \\
\text { балки } \\
{[9]}\end{array}$ \\
\hline$d$ & 0.16 & 0.18 & 1.1 & 0.95 \\
$r_{c}$ & 0.04 & 0.045 & 0.36 & 0.47 \\
$\delta$ & 0.08 & 0.095 & 0.21 & 0.69
\end{tabular}

3.4. Растяжение пластины с трещиной. Растяжение пластины с трещиной - известная задача, с которой началось развитие механики разрушения. Значения параметров расстояния по данным растяжения пластины с трещиной получим исходя из предположения равносильности нелокальных критериев и критерия Ирвина [10]. Значения параметров расстояния структурного критерия, критерия критической точки и градиентного критерия для данных работы [6] определяются соответственно по формулам

$$
\begin{aligned}
d & =\frac{2}{\pi}\left(\frac{K_{I c}}{\sigma_{c}}\right)^{2}, \\
r_{c} & =\frac{1}{2 \pi}\left(\frac{K_{I c}}{\sigma_{c}}\right)^{2}, \\
\delta & =\frac{1}{\pi}\left(\frac{K_{I c}}{\sigma_{c}}\right)^{2} .
\end{aligned}
$$

Значения характерных расстояний $d, r_{c}, \delta$, полученных на основе экспериментальных данных работы [6], приведены в таблице.

\section{4. Заключение}

В таблице приведены значения характерных расстояний $d, r_{c}, \delta$, определенные в разных схемах испытаний: с сингулярным концентратором напряжений, когда напряжение формально возрастает до бесконечности при приближении к концентратору напряжений (растяжение пластины с разрезом $\omega=\pi$ и угловым вырезом $\pi / 2<\omega<\pi)$; с регулярным концентратором напряжений (растяжение пластины с круглым отверстием и изгиб балки).

Значения параметров расстояния в задачах с одинаковым типом концентратора напряжений находятся в рамках одного порядка, и значения характерных расстояний в среднем тем больше, чем равномернее поле напряжений. В задаче с регулярным концентратором напряжений параметр $d$ в 6.9 раз больше, чем с сингулярным, $r_{c}-$ в 11.8 раз, $\delta$ - в 8.6 раз.

Результаты работы показывают, что как в случае с сингулярным концентратором напряжений, так и в случае с регулярным концентратором напряжений следует использовать нелокальные критерии разрушения. 
Отметим, что величина градиента напряжений в материале определяется как геометрическими особенностями конструкции, так и способом приложения нагрузки. Для инженерной практики актуальной потребностью является наличие стандартных программ, позволяющих проводить расчеты для различных конструкций и произвольных схем нагружения. Критерии разрушения, применяемые в компьютерных расчетах, должны, очевидно, использовать фиксированный набор параметров материала.

Анализ, однако, показывает, что существующие нелокальные критерии не являются в достаточной мере универсальными. Задачи с регулярным и сингулярным полем напряжения должны рассматриваться отдельно друг от друга. В рамках каждого из этих классов задач параметр, описывающий характерное расстояние, меняется мало, и, следовательно, в расчетах, соответствующих различным схемам испытаний, можно использовать нелокальный критерий с фиксированным набором параметров. В то же время для задач разных классов параметр расстояния существенно различается.

Следует отметить, что в реальных задачах различие между регулярными и сингулярными концентраторами является в значительной мере условным (пример вырез с малым радиусом закругления). Вопрос о возможной модификации нелокальных критериев, которая позволила бы в рамках единой модели с фиксированными параметрами проводить расчеты для полей напряжения с произвольной степенью неоднородности, остается открытым.

\section{Список литературы}

[1] L.P. Isupov, S.E. Mikhailov. Appl. Mech. 68, 597 (1998).

[2] P. J. E. Wright, F. Garwood. Mag. Concrete Res. 11, 67 (1952).

[3] Ю.В. Петров, А.А. Груздков, В.А. Братов. Физ. мезомеханика 15, 2, 15 (2012).

[4] D. Taylor. Struct. Durability Health Monitoring 2, 1 (2006).

[5] В.Д. Харлаб. Изв. вузов. Строительство и архитектура 11, 39 (1994).

[6] X.B. Zhang, J. Li. Eng. Fracture Mech. 75, 4925 (2008).

[7] S.P. Timoshenko, J.N. Goodier. Theory of elasticity. 3 ed. McGraw-Hill, N.Y. (1970). 522 p.

[8] Н.Ф. Морозов. Математические вопросы теории трещин. Наука, М. (1984). 256 c.

[9] F. Mujika, N. Carbajal, A. Arrese, I. Mondragon. Polymer Testing 25, 766, (2006).

[10] V.A. Bratov, N.F. Morozov, Yu.V. Petrov. Dynamic strength of continuum. St. Petersburg University Press, SPb. (2009). $223 \mathrm{p}$. 